\title{
Application of mathematical programming and modeling methods for monitoring the technical condition of underwater crossings of main gas pipelines
}

\author{
N.S. Pavlov ${ }^{1}$, B.Y. Vasilev ${ }^{1}$, and D.A. Bykasov ${ }^{1, *}$ \\ ${ }^{1}$ Saint Petersburg Mining University, 2, Line 21, Vasilievsky Ostrov,199106, Saint Petersburg, Russia
}

\begin{abstract}
The article deals with the problem of geodetic monitoring of the technical condition of underwater gas pipeline crossings. The theoretical scheme of increasing the accuracy of depth determination is studied in detail. In this paper, the goal is to increase the accuracy of obtaining the depth of the gas pipeline. The accuracy of the planned position for the largest survey scale (1:500) is 0.75 meters and is achieved using satellite equipment, even without the use of a dynamic positioning system, which allows, if necessary, to increase the accuracy of the data obtained by determining the orientation angles of the vessel. Despite the high popularity of this complex, the accuracy of depth measurements does not correspond to regulatory documents, which raises the question of the need to change the method of measurement and data processing, since the performance of work on monitoring the technical condition of gas pipelines is an important and relevant task. In order to control the developed methodology and automate measurement processing, two programs were written in the Python 3.7 programming language in the Spyder environment.
\end{abstract}

\section{Introduction}

Over the past thirty years, geodetic equipment has made a big leap forward. This rapid development of technology allowed surveyors to receive and process a huge amount of data about the objects under study. Thanks to the development of information technologies and the introduction of programming methods in geodetic production, it is possible to solve many problems using new methods, this idea is confirmed in the articles [1-9]. The application of information technologies in geodesy allows us to automate the calculation process in any software environment, which saves the final result from gross errors. And also, most importantly, it makes it possible to model solutions to various geodetic problems [10] associated with hazardous production, whether it is an executive survey when aligning crane tracks, or creating a model of the terrain of oil fields. Especially relevant today is the task of geodetic monitoring of the technical condition of the underwater crossing of gas pipelines, this is evidenced by the work of the authors [11-16]. The modern gas supply system on the

\footnotetext{
Corresponding author:bykasov.1996@mail.ru
} 
territory of the Russian Federation is a well-developed main gas pipeline network. Due to the large distances between them, the lengths of linear sections of pipelines reach hundreds or thousands of kilometers, which increases the wear of the system as a whole.

The systems used for monitoring the technical condition of underwater pipeline crossings use a combination of hydroacoustic and electromagnetic methods, which is currently the main and most accurate way to monitor the technical condition of a gas pipeline. Despite the high popularity of such complexes, the accuracy of depth measurements does not correspond to regulatory documents, which was calculated in [17]. According to [17], even at a depth of five meters, the instrument error exceeds the permissible one, which is described in the regulatory documentation. However, at the moment there are no devices and methods for measuring the depth of the gas pipeline at the underwater crossing, which could be used remotely and get a lower error. It is worth mentioning that most surveys performed by remote means are performed in a single pass along the tacks, which eliminates the appearance of sufficient redundancy of measurements. Redundancy of measurements increases the accuracy and plausibility of the obtained solutions, but with the increase in the amount of information received, the complexity of data processing also increases, as noted in articles [18] and [19]. There is a need to use computers with high performance characteristics in order to solve the task in a special software package. In order to obtain redundancy of measurements on the occurrence of the pipeline in the ground, repeated passes on tacks on water barriers are necessary. In addition, if one focuses on the profile of the pipeline (Fig. 1) at the underwater crossing, the laid pipe can not be described mathematically in its entirety, since it consists of sections with different curvature, which, when approximated, will give a large distortion and will not allow working with a single object. In figure 1, vertically elevation marks in meters are marked, and under the numbers: 1-ground marks (19.10.2016), 2 - pipe top marks (19.10.2016), 3 - water horizon (11.07.2018), 4 - ground marks (11.07.2018), 5 - pipe top marks (11.07.2018). In this regard, this article will focus on increasing the accuracy of obtaining the depth of the gas pipeline.

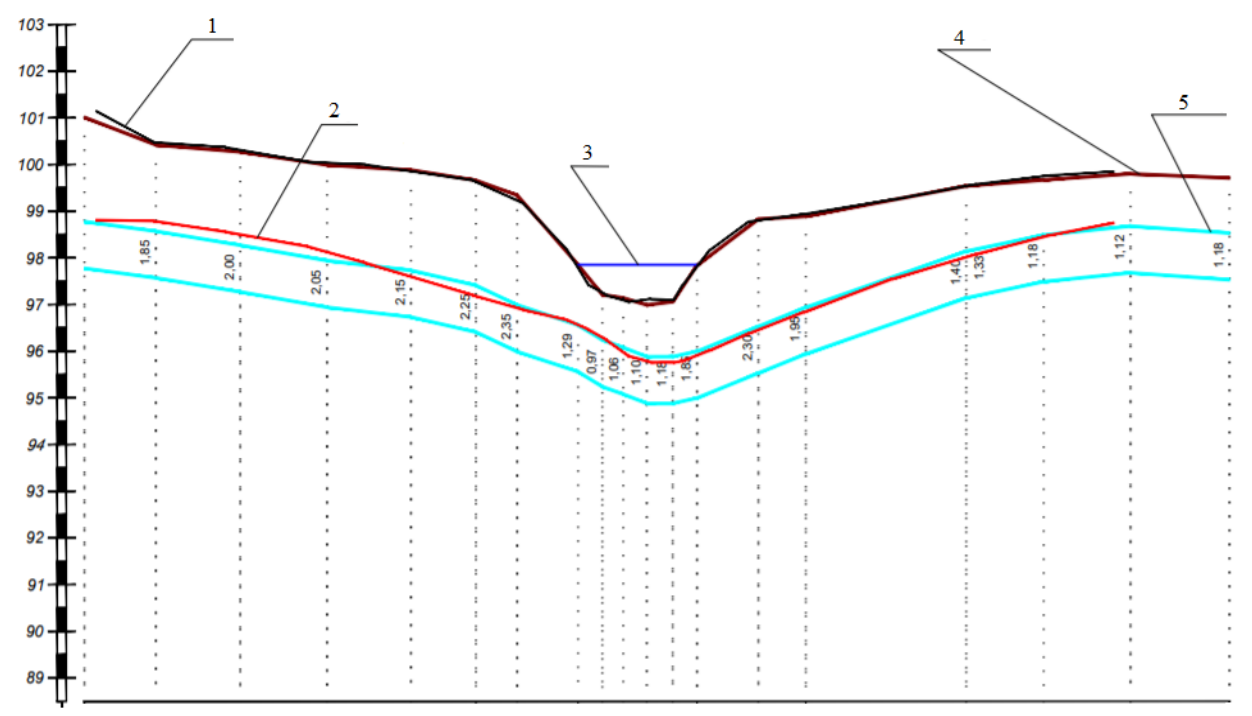

Fig. 1. The pipe profile.

\section{Materials and methods}


The ship's pipeline finder makes constant measurements with a frequency that depends on the speed of the ship. They try to keep the speed constant during all measurements in order to get the same measurement density. Due to the fact that the device is able to perform measurements with a fairly high frequency (on average, five points per meter), two points can fall on pipes with a diameter of half a meter, which leads to the appearance of measurements that are used in processing as additional ones. The depth is calculated from the section of the electromagnetic field, which is formed at some distance from the pipe. The resulting section has additional redundancy, but it is mostly an approximation. As the passport accuracy shows, this data may not be sufficient, so the only way to increase redundancy to increase accuracy by eliminating accidental instrument errors, as well as controlling gross errors, is to rerun one section of the underwater crossing. It is assumed that the data obtained from the second pass will have the necessary redundancy, which will at least reduce the impact of the random part of the instrument error by bringing them to the average, and also increase the controllability.

The use of rerunning should reduce the effect of multidirectional instrument error, but without reference measurements, the data obtained cannot be considered reliable and meet regulatory requirements. To obtain reference measurements, there is only one method of measurement - a puncture by a diver during a dive. Since diving descents are complex and expensive, it is necessary to perform them only after performing preliminary processing of data obtained by remote methods, and also try to minimize the number of such descents, limiting them to areas with exposure or a critical thickness of the protective layer above the pipeline. Based on the foregoing, it is recommended to perform reference measurements at the depth of the pipeline more than five meters from the water's edge; underoccurence of the pipeline in the soil of the channel of the water obstacle over 0.5 meters; in places where there is assumed to be anomalies affecting the measurements; to perform at least three reference measurements with distance, which should not be less than specified in the instructions to these works; it is desirable to perform measurements at different depths but starting from five meters; if possible to combine areas of denudation and sagging with surveying measurements in order to minimize the number of dives; not to use for the calculation of the correction measurements made during diving descents in anomalous zones when a deviation is detected exceeding double instrumental error.

It should be noted that with a significant thickness of the protective roller (more than three meters), the depth of the pipeline, even if obtained with an instrument error of more than ten centimeters, does not affect the main purpose of monitoring. Namely, establishing the fact that the pipe is protected from mechanical damage, and since, according to the regulatory documentation, the thickness of the protective roller should not be less than one meter, the task can be considered completed. However, in order to comply with the regulatory documentation, the elimination of all possible errors is considered a necessary task.

\section{Obtained results}

Let us consider the processing of the data obtained as a result of measurements with the AMC "SKAT-TIEM2/2A" in two passes in the PipeTracer software. AMC "SKAT-TIEM2/2A" was developed and implemented by the research and production enterprise "Fort XXI". Both passes were processed independently of each other. Since the depth of the gas pipeline does not exceed five meters along the entire length of the underwater passage, it is considered impractical to conduct additional measurements and diving descents in order to obtain reference measurements. The error obtained by a single pass, in this case, is within the limits set by the regulatory documentation. Therefore, as test data, we used the results obtained under the condition that the pipeline depth at the underwater crossing exceeds five meters. 
The following sequence of processing the received data is proposed:

Independent processing of data of two passes in the program, in this case PipeTracer, with interpolation and obtaining data on the depth of occurrence in the same places of the gas pipeline section. It is assumed that at this stage, part of the random instrument error is eliminated using algorithms for internal processing of electromagnetic field sections, and also some gross errors are cut off by excluding them from further processing.

Uploading processing results to PipeTracer software in Excel, as well as adding data about underwater measurements (spatial coordinates and depth of occurrence). Data on the depth of the gas pipeline in the places of reference measurements should be obtained from the program.

Averaging the depth of occurrence obtained over two passes, for additional control and reducing the influence of random instrument error, using the formula (1):

$$
h_{m}=\frac{h_{1}+h_{2}}{2}
$$

where $h_{m}$ - is the average value of the depth of occurrence; $h_{1}$ - is the depth of the gas pipeline obtained in the first pass; $h_{2}$ - is the depth of the gas pipeline obtained in the second pass.

Calculation of instrument errors for each measurement, according to the passport accuracy of the device. In this case, TIEM-2 was used, and the depth of the gas pipeline did not exceed ten meters, so the formula for determining the instrument error is as follows (2):

$$
m=0,01 \cdot h_{m}+0,05
$$

Calculation of the three amendments $v_{i t}$ at reference measurement locations (3):

$$
v_{i t}=h_{i t}-h_{m}
$$

where $h_{i t}$ - is the depth of the gas pipeline obtained as a result of reference measurements. Since it is assumed that the random part of the instrument error is significantly reduced in the previous stages, the reference measurements should be of the same sign, that is, show the part of the systematic error that was not excluded during the program processing of two independent passes.

Calculating of the average amendment $v_{i t m}$ using the formula (4):

$$
v_{i t m}=\frac{v_{i t 1}+v_{i t 2}+\ldots+v_{i t n}}{n}
$$

where $n-$ is the number of reference measurements.

Calculation of the average depth $h_{i t m}$ of occurrence in the performed reference measurements (5):

$$
h_{i t m}=\frac{h_{i t 1}+h_{i t 2}+\ldots+h_{i t n}}{n}
$$

Calculating the maximum amendment $v_{\max }$, reduced to the ten-meter mark (6): 


$$
v_{i t m}=\frac{10}{h_{i t m_{\max }}}
$$

To introduce the resulting correction to the measurement, the inverse weight $p$ must be calculated using the formula (7):

$$
\frac{1}{p}=\left(1-\frac{0,01}{m_{i s m}}\right) \cdot k_{g r} \cdot k_{k t b} \cdot k_{g l u}
$$

where $k_{g r}$ - is ground coefficient; $k_{k t b}$ - is coefficient of the number of pipes on the crossing; $k_{g l u}$ - is depth coefficient; $m_{i s m}$ — instrument error of each measurement.

Then it is necessary to calculate to calculate the corrected value of the depth of the gas pipeline pipe using the formula (8):

$$
h_{i s p}=h_{i t m}-v \cdot \frac{1}{p_{\max }}
$$

An indicator of the residual theoretical instrument error $m_{\text {ost }}$ is the difference between the amendment and the instrument mean square error (9):

$$
m_{o s t}=h_{i s p}-h_{i t m}
$$

Since it was not possible to obtain data on underwater river crossings with a pipeline depth exceeding ten meters, simulated measurements were used as test data, which reflect all the key boundaries of increasing depth. The coefficient value $k_{k t b}$ depends on the distance between the pipes at the underwater crossing, as well as on their number and location. The proposed options are shown in Table 1.

Table 1. Coefficient $k_{k t b}$ depending on the number of pipes at the crossing.

\begin{tabular}{|l|c|}
\hline \multicolumn{1}{|c|}{ Configuration of pipes in the area of the underwater crossing } & Value of coefficient $k_{k t b}$ \\
\hline One pipe on the crossing & 0.80 \\
\hline There is one pipe nearby (a distance of more than 30 meters) & 0.85 \\
\hline There are two pipes nearby (a distance to both is more than 30 meters) & 0.90 \\
\hline There is one pipe nearby (a distance of less than 30 meters) & 0.95 \\
\hline $\begin{array}{l}\text { There are two pipes nearby (the distance to one is more than 30 meters. } \\
\text { and to the other is less than 30 meters) }\end{array}$ & 0.97 \\
\hline There are two pipes nearby (both less than 30 meters away) & 1.00 \\
\hline
\end{tabular}


In order to control the developed measurement processing technique, two programs were written in the Python 3.7 programming language in the Spyder environment. The Python 3.7 programming language was chosen because it is most convenient for writing software modules and is most in demand among surveyors in production, since it solves many problems [20-23]. The main goal of the first program is to check the correctness of calculations performed in Microsoft Excel, as well as to speed up the data processing process. The goal of the second program is to increase automation, greater versatility, and a different set of simulated second pass data to track the effectiveness of the technique when changing a number of parameters (differences in passes and reference measurements). The first block of the first program, shown in Figure 2, is necessary for entering the source data and the necessary software libraries: coefficients; initial depths of occurrence; reference measurements, etc. For further work with the data, preliminary calculations of the amendment were performed, which was calculated from reference measurements.

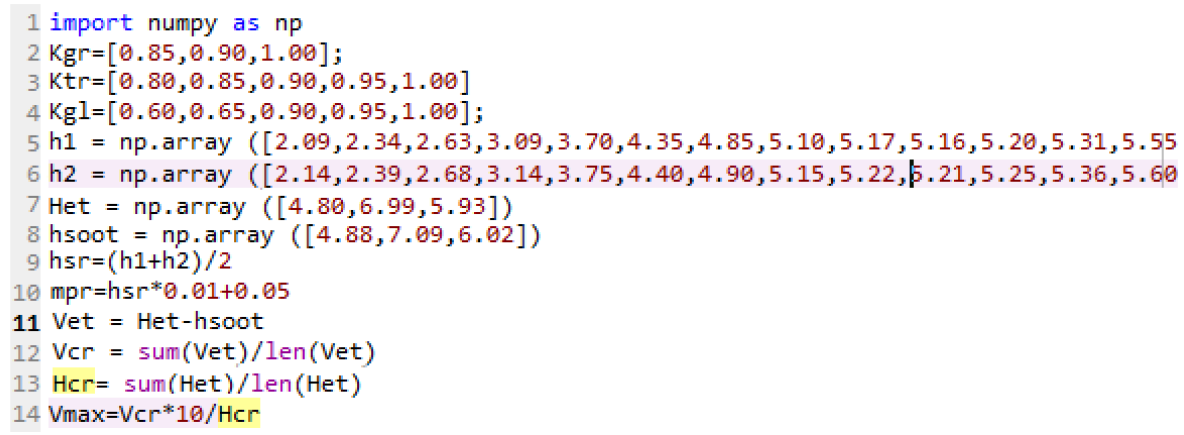

Fig. 2. The original data and the preliminary calculation.

The final stage of the program is to calculate the final height, as well as the instrument and residual error, which as a result correspond to those calculated using Microsoft Excel. Part of the calculation algorithm is shown in Figure 3. 


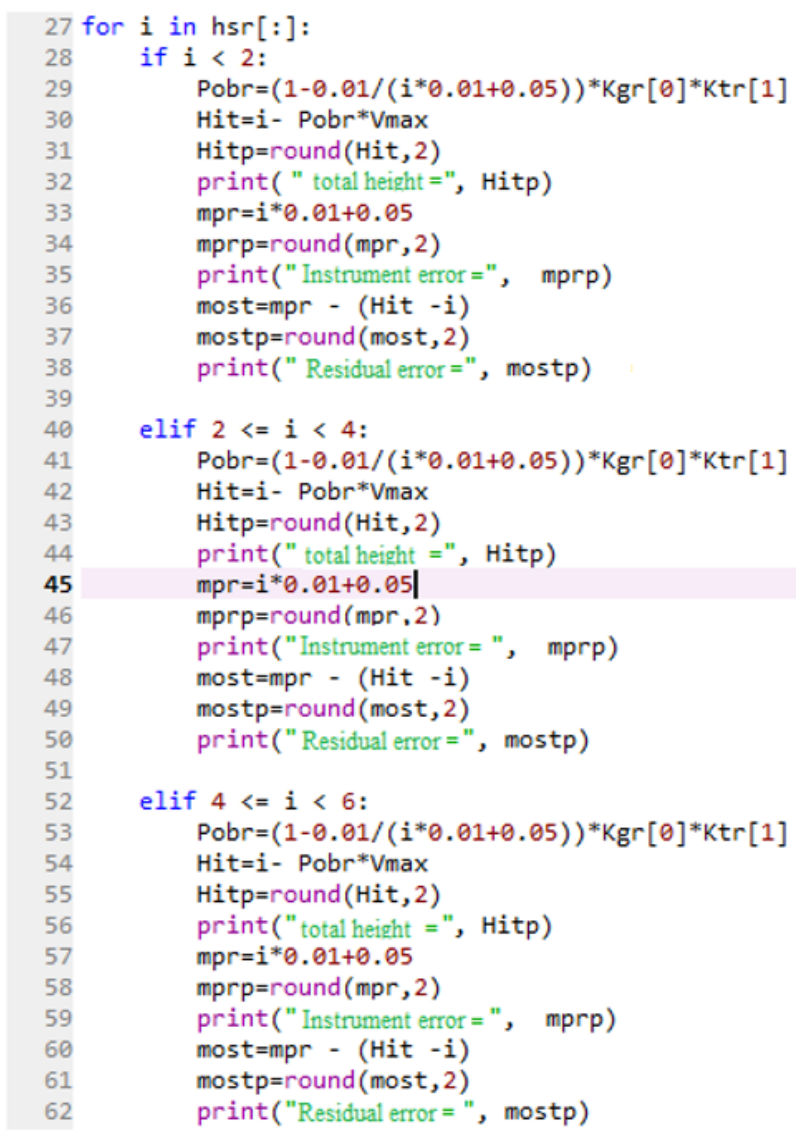

Fig. 3. The final calculations.

A fragment with the results of processing full-scale measurements using the program is shown in Figure 4. All heights and additional calculations are recorded in the same order as the original data, which makes it easy to add coordinates to them if necessary. This program is a lightweight version of the second one, since it requires entering the source data manually, but it is easier to write and implement.

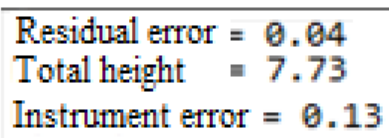

Fig. 4. Results of the program.

The first block of the second program is loading a specialized library for working with databases (pandas), as well as introducing coefficients necessary for further processing. This step is shown in Figure 5. The second block is loading survey data in .csv format over two passes processed in the PipeTracer program, as well as reference measurements. An example of the source data is shown in Figure 5. For further data processing, array sections were performed in the height column, as well as data transfer from DataFrame to the usual lists. 


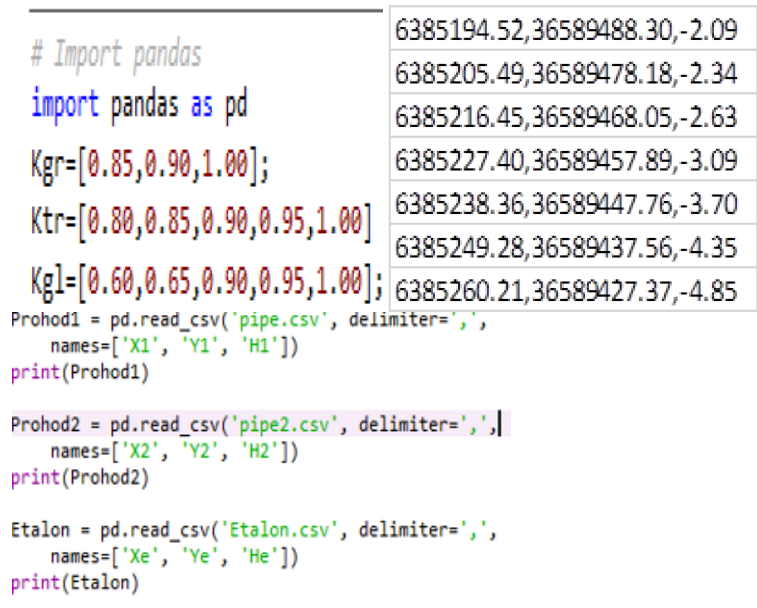

a $\mathrm{c}$

Fig. 5. Structure of the second program: a - is preparatory stage; $b$ - is sample source data; $c$ - is loading source data.

An important step in automating data processing was to find matches between the average elevation values and reference measurements by coordinates, as shown in Figure 6. Thanks to the built - in function for finding dependencies in DataFrame (merge), there is no need to search and compare matches manually.

\section{hst. merge(het, how=' inner')}

Fig. 6. Automation of matching.

The further processing algorithm does not differ from the first program, since it does not require additional actions on the part of the user.

\section{Discussion}

As a result of processing the test data, the residual value of the instrument error did not exceed five centimeters, which makes it possible to use this scheme for processing data on the survey of underwater gas pipeline crossings at depths of up to ten meters. Thus, a balanced solution is proposed to reduce the influence of instrument error on sections of underwater crossings with a depth of more than five meters due to unacceptable instrument error. The modified method of performing field work, as well as additional processing of measurement results, will allow monitoring underwater crossings of the main gas pipeline with greater accuracy and control.

According to the results of the work, it can be seen that the method is stable when modeling other data, which favorably affects the theoretical foundations laid in the idea of improving the accuracy and controllability of the results of geodetic monitoring of the technical condition of the main gas pipeline at an underwater crossing.

\section{Conclusions}

Changes in the method of work and the method of processing the data obtained during the survey of underwater crossings of main gas pipelines should theoretically lead to an increase 
in the accuracy of measurements by reducing the influence of random and systematic errors, including instrument errors, as well as to additional controllability. The set of proposed innovations, taking into account the complexity of measurements, takes into account various factors that affect the reliability of the data obtained, and represents a combination of reducing the influence of random instrument error and introducing additional amendments from reference measurements to further reduce the influence of random error and eliminate systematic errors. As a result, a program was developed that can automatically process data received from Pipetacer software without user intervention. High processing speed and the absence of errors related to the human factor make it possible to perform the proposed processing method more efficiently and reliably. In the future, it is planned to process new production data for debugging the program.

\section{References}

1. Retscher G, Mentes G, and Reiterer A 2014 Inter. Association of Geodesy Symposia 139481

2. Shi S, Shi Y and Zhao W 2018 Wuhan Daxue Xuebao (Xinxi Kexue Ban)/Geomatics and Information Science of Wuhan University 43(5) 779

3. Leandro R F, da Silva C A U, Segantine P C L, and Santos M C 2007 GPS World 18(9) 60

4. Kuzin A A, Valkov V A and Kazantsev A I 2018 JP Conf. Series 1118(1) 012022

5. Kochneva A A and Kazantsev A I 2017 J. Ind. Pollut. Control 33(1) 1000

6. Men'Shikov S N, Dzhaljabov A A, Vasiliev G G, Leonovich I A and Ermilov O M 2019 Journal of Mining Institute 238430

7. Kiani M 2020 Geodesy and Geodynamics 11(5) 376

8. Zeng H, Chang G, He H and Li K 2020 Earth, Planets and Space 72(1)

9. Mazurov B T, Mustafin M G and Panzhin A A 2019 Journal of Mining Institute 238 376

10. Tang F, Lu J, Wei S, Li X, He K and Yang Q 2020 Meitan Xuebao/Journal of the China Coal Society 45(7) 2655

11. Thiberville C, Wang Y, Waltrich P, Williams W, and Kam S I 2019 Society of Petroleum Engineers - SPE Gas and Oil Technology Showcase and Conference 2019

12. Lin G, Zhoumo Z, Xinjing H, Mingze L, Hao F, Jian L and Xiaobo R 2020 IEEE Access 833926

13. Grythe K, Jensen I A and Knudsen O O 2020 14th European Conference on Antennas and Propagation, EuCAP 2020

14. Pnev A B, Zhirnov A A, Stepanov K V, Nesterov E T, Shelestov D A and Karasik V E 2015 Paper presented at the Journal of Physics: Conference Series 584(1)

15. Barbagelata L and Kostianoy A G 2016 Subsea leak detection with passive acoustic technology Co.L.mar

16. Ilyushin Y V and Afanaseva O V 2019 Journal of Mining Institute 240686

17. Pavlov N S 2015 Tekhnologii geodezicheskogo obespecheniya obsledovaniy podvodnykh perekhodov magistral'nykh gazoprovodov (PhD Thesis) (Saint-Petersburg: Saint Petersburg mining University)

18. Liu G H and Feng Q Y 2009 ICIC 20092349 
19. Suzuki T, Takahashi Y and Amano Y 2016 ION GNSS 201621455

20. Martens H R, Rivera L and Simons M 2019 Earth and Space Science 6(2) 311

21. Zrinjski M, Barković $Đ$ and Puklavec I 2014 International Multidisciplinary Scientific GeoConference Surveying Geology and Mining Ecology Management 2(2) 443

22. Nielsen J, Tscherning C C, Jansson T R N and Forsberg R 2012 International Association of Geodesy Symposia 136443

23. Weigel M, Preuss T, and Brüstel J 2010 CISIS 2010 - the 4th International Conference on Complex, Intelligent and Software Intensive Systems 544 